\title{
Toxicological Studies of Mulberry Powdery Mildew Effective Fungicide Residues on Growth and Development of Silkworm (Bombyx mori L.), Cocoon and Silk Quality Parameters
}

\author{
S.E. Manjunatha, V.B. Sanath Kumar* and N. Kiran Kumar \\ Department of plant pathology, College of Agriculture, V.C. Farm, \\ Mandya- 571405, Karnataka, India \\ *Corresponding author
}

\author{
A B S T R A C T
}

\begin{tabular}{|l|}
\hline K e y w o r d s \\
Powdery mildew, \\
Silkworm (Bombyx \\
mori L.), Toxicity, \\
Fungicides, Cocoon, \\
Denier.
\end{tabular}

Study on assessing the toxic effects of fungicides on silkworm (Bombyx mori L.) and also on cocoon and silk quality parameters revealed that carbendazim 50\% WP and wettable sulphur $80 \% \mathrm{WP}$ at $0.1 \%$ were found to have no toxic effect by showing zero larval mortality at three days after treatment. The larval weight and larval length of $29.33 \mathrm{~g}$ and $5.73 \mathrm{~cm}$ respectively in carbendazim 50\% WP and $29.10 \mathrm{~g}$ and $5.42 \mathrm{~cm}$ ) in wettable sulphur $80 \%$ WP was found to be higher than other fungicidal treatments. The cocoon characters like cocoon weight of $11.37 \mathrm{~g}$ and $11.22 \mathrm{~g}$, pupal weight of $8.40 \mathrm{~g}$ and $8.29 \mathrm{~g}$, shell weight of $2.95 \mathrm{~g}$ and $2.93 \mathrm{~g}$, shell ratio of 26.12 and 26.11 per cent and cocoon yield of $398.34 \mathrm{~g} / \mathrm{dfl}$ and $392.70 \mathrm{~g} / \mathrm{dfl}$ was higher in carbendazim $50 \% \mathrm{WP}$ and wettable sulphur $80 \% \mathrm{WP}$ at 0.1 per cent concentration respectively. The silk quality traits like filament length of $857.02 \mathrm{~m}$ and $820.58 \mathrm{~m}$, filament weight of $0.20 \mathrm{~g}$ and $0.21 \mathrm{~g}$ and denier of 2.14 $\%$ and $2.27 \%$ was found to be higher in carbendazim 50\% WP and wettable sulphur $80 \%$ WP treatment at 0.1 concentration.

\section{Introduction}

Mulberry (Morus alba) is a perennial plant belongs to the family Moraceae, the food plant of silkworm (Bombyx mori L.). It is cultivated in both tropical and temperate countries of the world. Though mulberry cultivation is practiced in various climates in India, it is extensively grown in the tropical zone covering Karnataka, Andhra Pradesh and Tamil Nadu states with about 90 percent of area where, most of the sericulture industry is concentrated. In the sub-tropical zone, West Bengal, Himachal Pradesh and the north eastern states have major areas under mulberry cultivation (Datta, 2011). Among the major diseases occurring on the mulberry powdery mildew caused by Phyllactinia corylea causes 5-10\% loss due to defoliation and an additional loss of $20-25 \%$ through destruction of leaf area (Sukumar and Ramalingam, 1989; Teotia and Sen, 1994). Feeding the diseased leaves affect the growth and development of silkworm. The disease is managed by spraying systemic fungicides. Since, the mulberry leaf is fed directly to the silkworms and as the worms are highly fragile, the fungicidal spray residues affect the health and cocoon quality and weight. Also, the information on the residual toxicity of fungicides on silkworm is little and inadequate. Hence, there is a need to screen 
the fungicides for selection of suitable, effective and safer compounds for control of diseases in mulberry and the present study undertaken is in this direction.

\section{Materials and Methods}

Toxicological studies on silkworm (Bombyx mori L.) Cross breed hybrid (Pure Mysore $\mathrm{x}$ CSR2) was carried out Department of Plant Pathology, College of Agriculture, V.C. Farm, Mandya. Different concentrations of fungicides viz., $\mathrm{T}_{1}, \mathrm{~T}_{2}, \mathrm{~T}_{3}$ (Carbendazim $50 \%$ $\mathrm{WP}$ at $0.1,0.2$ and $0.3 \%$ concentration); $\mathrm{T}_{4}$, $\mathrm{T}_{5}, \mathrm{~T}_{6}$ (Wettable sulphur $80 \% \mathrm{WP}$ at $0.1,0.2$ and $0.3 \%$ concentration); $\mathrm{T}_{7}, \mathrm{~T}_{8}, \quad \mathrm{~T}_{9}$ (Tebuconazole 50\%+Trifloxystrobin $25 \% \mathrm{WG}$ at $0.1,0.2$ and $0.3 \%$ concentration); $\mathrm{T}_{10}, \mathrm{~T}_{11}$, $\mathrm{T}_{12}$ (Carbendazim 12\%+ Mancozeb 63\% WP at $0.1,0.2$ and $0.3 \%$ concentration); $\mathrm{T}_{13}, \mathrm{~T}_{14}$, $\mathrm{T}_{15}$ (Hexaconazole 5\% $\mathrm{EC}$ at $0.1,0.2$ and $0.3 \% \quad$ concentration); $\quad \mathrm{T}_{16}, \quad \mathrm{~T}_{17}, \quad \mathrm{~T}_{18}$ (Difenconazole 25\% EC at $0.1,0.2$ and $0.3 \%$ concentration); $\mathrm{T}_{19}, \mathrm{~T}_{20}, \mathrm{~T}_{21}$ (Mancozeb $75 \%$ $\mathrm{WP}$ at $0.1,0.2$ and $0.3 \%$ concentration); $\mathrm{T}_{22}$ Control (Water spray) were prepared by w/v and $\mathrm{w} / \mathrm{w}$ and the mulberry leaves were immersed in respective aqueous solutions for 2-3 minutes to absorb the solution uniformly. Later, the leaves were shade dried for 30 seconds, the treated leaves were fed once to $3^{\text {rd }}$ instar $1^{\text {st }}$ day silkworm larvae. After treatment imposition, fresh untreated leaves were offered until final day of $5^{\text {th }}$ instar. Leaves sprayed with water and fed to silkworms served as control. Treatments were replicated thrice by using twenty worms with the statistical design of CRD.

\section{Results and Discussion}

\section{Fungicide residue effect on larval mortality}

The observations of fungicidal toxicity on larval mortality were recorded during $3^{\text {rd }}$ instar stage at 1, 2 and 3 days after treatment
(DAT). In the present investigation it was observed that all the fungicides showed toxic effects on silkworm larvae at $1^{\text {st }}$ and $2^{\text {nd }}$ days after treatment. However, the toxic effects were gradually reduced at 3 days after treatment in some fungicides at different concentrations.

First day after treatment (DAT), it was noticed that the maximum larval mortality $(31.89 \%)$ was recorded in tebuconazole $50 \%+$ trifloxystrobin $25 \% \mathrm{WG}$ at $0.3 \%$ which is on par with carbendazim $12 \%+$ mancozeb $63 \%$ WP $(30.80 \%)$ at $0.3 \%$. This was followed by hexaconazole 5\% EC $(23.20 \%)$ at $0.3 \%$, mancozeb $75 \% \mathrm{WP}(22.93 \%)$ at $0.3 \%$. The zero per cent larval mortality was observed in control treatment and least larval mortality of 4.95 per cent was recorded in wettable sulphur $80 \% \mathrm{WP}$ at 0.1 per cent which was on par with carbendazim $50 \%$ WP $(4.99 \%)$ at 0.1 per cent concentration. The data is presented in Table 1.

At second DAT, it was found that the maximum larval mortality $(20.78 \%)$ was recorded in tebuconazole $50 \%+$ trifloxystrobin $25 \% \mathrm{WG}$ at $0.3 \%$ which is on par with mancozeb $75 \%$ WP (19.93\%) at $0.3 \%$ and tebuconazole $50 \%+$ trifloxystrobin $25 \% \mathrm{WG}(19.48 \%)$ at 0.2 per cent concentration. The lowest larval mortality $(0.00 \%)$ was observed in control treatment and it was followed by wettable sulphur $80 \%$ WP $(4.00 \%)$ at $0.1 \%$ which is on par with carbendazim 50\% WP (4.02\%) at 0.1 per cent concentration and other treatments showed a significant effect on larval mortality, with mancozeb $75 \%$ WP (19.93\%) at $0.3 \%$ and tebuconazole $50 \%+$ trifloxystrobin $25 \%$ WG $(19.48 \%)$ at 0.2 per cent concentration. The zero per cent larval mortality was observed in control treatment and it was followed by wettable sulphur $80 \%$ WP $(4.00 \%)$ at $0.1 \%$ which was on par with carbendazim 50\% WP $(4.02 \%)$ at 0.1 per cent concentration and 
other treatments showed a significant effect on larval mortality.

Three DAT the maximum larval mortality (16.65\%) was recorded in tebuconazole 50\%+ trifloxystrobin $25 \% \mathrm{WG}$ at $0.3 \%$ which was statistically significant over other treatments during three days after treatment (DAT). This was followed by carbendazim $12 \%+$ mancozeb $63 \%$ WP $(13.95 \%)$ at 0.3 per cent concentration and hexaconazole $5 \%$ EC with $12.04 \%$ at $0.3 \%$ and 11.36 per cent with mancozeb $75 \% \mathrm{WP}$ at $0.3 \%$. The zero per cent larval mortality was observed in control treatment, wettable sulphur $80 \% \mathrm{WP}$ at $0.1 \%$ and carbendazim $50 \% \mathrm{WP}$ at $0.1 \%$ which were statistically significant when compared with other treatments. Similar results were reported by Sikdar et al., (1979) and Aherkar et al., (1995).

\section{Fungicide residue effect on larval weight}

The observations of fungicidal toxicity on larval weight were recorded during $5^{\text {th }}, 6^{\text {th }}$ and $7^{\text {th }}$ day of $5^{\text {th }}$ instar, and it was found that there was a significant difference between treatments. The results from the Table 2 revealed that all the seven fungicides used at three different concentrations showed a significant effect on larval weight during $5^{\text {th }}$ day of $5^{\text {th }}$ instar. The highest larval weight $(20.30 \mathrm{~g})$ was recorded in control treatment which is at par with carbendazim 50\% WP with $18.85 \mathrm{~g}$ at 0.1 per cent concentration, followed by wettable sulphur $80 \%$ WP with $18.73 \mathrm{~g}$ at $0.1 \%$. These treatments were statistically significant over other treatments.

The lowest larval weight (14.56g) was recorded in tebuconazole $50 \%+$ trifloxystrobin $25 \% \mathrm{WG}$ at $0.3 \%$ which is on par with hexaconazole 5\% EC (14.60g) at 0.3 per cent concentration followed by mancozeb $75 \%$ WP (14.87g) at 0.3 per cent concentration.
At $6^{\text {th }}$ day of $5^{\text {th }}$ instar it was found that the maximum larval weight $(24.94 \mathrm{~g})$ was noted in control treatment which was on par with carbendazim $50 \% \mathrm{WP}(23.78 \mathrm{~g})$ at $0.1 \%$ followed by wettable sulphur $80 \%$ WP $(23.42 \mathrm{~g})$ at $0.1 \%$ and hexaconazole $5 \%$ EC $(22.30 \mathrm{~g})$ at $0.1 \%$. The lowest larval weight $(19.30 \mathrm{~g})$ was observed in tebuconazole $50 \%+$ trifloxystrobin $25 \% \mathrm{WG}$ at $0.3 \%$ followed by carbendazim 12\%+ mancozeb $63 \%$ WP $(19.32 \mathrm{~g})$ at 0.3 per cent concentration (Table 2).

During $7^{\text {th }}$ day of $5^{\text {th }}$ instar it was noticed that the highest larval weight (30.62g) was recorded in control treatment which is found on par with carbendazim 50\% WP (29.33g) at $0.1 \%$ followed by $(29.10 \mathrm{~g})$ with wettable sulphur $80 \%$ WP at $0.1 \%$.

However, the lowest larval weight of $21.80 \mathrm{~g}$ was found in tebuconazole $50 \%+$ trifloxystrobin $25 \% \mathrm{WG}$ at $0.3 \%$ followed by carbendazim 12\%+ mancozeb $63 \%$ WP $(21.97 \mathrm{~g})$ at $0.3 \%$ and mancozeb $75 \% \mathrm{WP}$ $(22.81 \mathrm{~g})$ at $0.3 \%$. It may be due to non-toxic effect of fungicides on growth and development of silkworm from 3 days after treatment. Similar results were obtained by Gayathri et al., (2011)

\section{Fungicide residue effect on larval length}

The observations of fungicidal toxicity on larval length was recorded during $5^{\text {th }}, 6^{\text {th }}$ and $7^{\text {th }}$ day of $5^{\text {th }}$ instar and found that there was a significant difference between treatments. During $5^{\text {th }}$ instar, $5^{\text {th }}$ day it was found that the larval length in control treatment, carbendazim $50 \% \mathrm{WP}$ treated at $0.1 \%$, carbendazim $50 \% \mathrm{WP}$ at $0.2 \%$, wettable sulphur $80 \% \mathrm{WP}$ at $0.1 \%$ and wettable sulphur $80 \% \mathrm{WP}$ at $0.2 \%$ has no significant difference when compared to other treatments with $4.85,4.784 .75,4.73$ and $4.70 \mathrm{~cm}$ respectively. 
Table.1 Effect of powdery mildew effective fungicides on larval mortality of silkworm (Bombyx mori L.)

\begin{tabular}{|c|c|c|c|c|c|}
\hline \multirow{2}{*}{$\mathbf{T r}$} & \multirow{2}{*}{ Fungicides } & \multirow{2}{*}{ Conc. (\%) } & \multicolumn{3}{|c|}{ Larval mortality (\%) } \\
\hline & & & 1 DAT & 2 DAT & 3 DAT \\
\hline $\mathrm{T}_{1}$ & Carbendazim50\% WP & 0.1 & $4.99(12.91)$ & $4.02(11.56)$ & $0.00(0.00)$ \\
\hline $\mathrm{T}_{2}$ & Carbendazim50\% WP & 0.2 & $10.02(18.45)$ & $8.50(16.95)$ & $4.95(12.85)$ \\
\hline $\mathrm{T}_{3}$ & Carbendazim50\% WP & 0.3 & $12.11(20.36)$ & $10.02(18.45)$ & $4.99(12.91)$ \\
\hline $\mathrm{T}_{4}$ & Wettable sulphur $80 \%$ WP & 0.1 & $4.95(12.85)$ & $4.00(11.54)$ & $0.00(0.00)$ \\
\hline $\mathrm{T}_{5}$ & Wettable sulphur $80 \%$ WP & 0.2 & $12.22(20.46)$ & $10.02(18.45)$ & $4.59(12.37)$ \\
\hline $\mathrm{T}_{6}$ & Wettable sulphur $80 \%$ WP & 0.3 & $16.00(23.58)$ & $14.90(22.70)$ & $4.96(12.87)$ \\
\hline $\mathrm{T}_{7}$ & $\begin{array}{l}\text { Tebuconazole 50\%+Trifloxystrobin } 25 \% \\
\text { WG }\end{array}$ & 0.1 & $19.02(25.85)$ & $12.00(20.27)$ & $4.99(12.91)$ \\
\hline $\mathrm{T}_{8}$ & $\begin{array}{l}\text { Tebuconazole 50\%+Trifloxystrobin } 25 \% \\
\text { WG }\end{array}$ & 0.2 & $20.07(26.61)$ & $19.48(26.19)$ & $8.00(16.43)$ \\
\hline $\mathrm{T}_{9}$ & $\begin{array}{l}\text { Tebuconazole 50\%+Trifloxystrobin } 25 \% \\
\text { WG }\end{array}$ & 0.3 & $31.89(34.38)$ & $20.78(27.12)$ & $16.65(24.08)$ \\
\hline $\mathrm{T}_{10}$ & Carbendazim 12\% + Mancozeb 63\% WP & 0.1 & $10.00(18.43)$ & $12.23(20.47)$ & $4.90(12.79)$ \\
\hline $\mathrm{T}_{11}$ & Carbendazim 12\% + Mancozeb 63\% WP & 0.2 & $21.17(27.39)$ & $16.96(24.32)$ & $9.97(18.40)$ \\
\hline $\mathrm{T}_{12}$ & Carbendazim 12\% + Mancozeb 63\% WP & 0.3 & $30.80(33.70)$ & $17.86(25.00)$ & $13.95(21.93)$ \\
\hline $\mathrm{T}_{13}$ & Hexaconazole 5\% EC & 0.1 & $9.47(17.92)$ & $9.13(17.59)$ & $5.18(13.15)$ \\
\hline $\mathrm{T}_{14}$ & Hexaconazole 5\% EC & 0.2 & $12.85(21.00)$ & $17.82(24.97)$ & $10.01(18.44)$ \\
\hline $\mathrm{T}_{15}$ & Hexaconazole 5\% EC & 0.3 & $23.20(28.79)$ & $16.90(24.27)$ & $12.04(20.30)$ \\
\hline $\mathrm{T}_{16}$ & Difenconazole $25 \%$ EC & 0.1 & $10.37(18.78)$ & $9.33(17.78)$ & $6.24(14.46)$ \\
\hline $\mathrm{T}_{17}$ & Difenconazole 25\% EC & 0.2 & $12.41(20.62)$ & $12.22(20.46)$ & $9.27(17.72)$ \\
\hline $\mathrm{T}_{18}$ & Difenconazole 25\% EC & 0.3 & $16.00(23.58)$ & $16.01(23.58)$ & $10.07(18.50)$ \\
\hline $\mathrm{T}_{19}$ & Mancozeb 75\% WP & 0.1 & $16.94(24.30)$ & $8.29(16.73)$ & $4.99(12.91)$ \\
\hline $\mathrm{T}_{20}$ & Mancozeb 75\% WP & 0.2 & $19.00(25.84)$ & $13.07(21.19)$ & $10.01(18.44)$ \\
\hline $\mathrm{T}_{21}$ & Mancozeb 75\% WP & 0.3 & $22.93(28.61)$ & $19.93(26.51)$ & $11.36(19.69)$ \\
\hline \multirow[t]{3}{*}{$\mathrm{T}_{22}$} & Control (Water spray) & - & $0.00(0.00)$ & $0.00(0.00)$ & $0.00(0.00)$ \\
\hline & \multicolumn{2}{|l|}{ S.Em \pm} & 0.69 & 0.57 & 0.31 \\
\hline & \multicolumn{2}{|l|}{$\mathrm{CD} @ \mathbf{P}=\mathbf{0 . 0 5}$} & 1.98 & 1.61 & 0.87 \\
\hline
\end{tabular}

$\mathrm{Tr}=$ Treatment; DAT = Days after treatment; Figures in the parenthesis are Arc sine transformed values; 
Table.2 Toxic Effect of different fungicides on larval weight and larval length of silkworm (Bombyx mori L.)

\begin{tabular}{|c|c|c|c|c|c|c|c|c|}
\hline \multirow[b]{2}{*}{$\mathbf{T r}$} & \multirow[t]{2}{*}{ Fungicides } & \multirow{2}{*}{$\begin{array}{c}\text { Conc. } \\
(\%)\end{array}$} & \multicolumn{3}{|c|}{$\begin{array}{c}\text { Average larval weight (g) } \\
\text { at } 5^{\text {th }} \text { instar }\end{array}$} & \multicolumn{3}{|c|}{$\begin{array}{c}\text { Average larval length }(\mathrm{cm}) \\
\text { at } 5^{\text {th }} \text { instar }\end{array}$} \\
\hline & & & $5^{\text {th }}$ day & $6^{\text {th }}$ day & $7^{\text {th }}$ day & $5^{\text {th }}$ day & $6^{\text {th }}$ day & $7^{\text {th }}$ day \\
\hline $\mathrm{T}_{1}$ & Carbendazim50\% WP & 0.1 & 18.85 & 23.78 & 29.33 & 4.78 & 5.45 & 5.73 \\
\hline $\mathrm{T}_{2}$ & Carbendazim50\% WP & 0.2 & 16.57 & 20.70 & 25.63 & 4.75 & 5.33 & 5.42 \\
\hline $\mathrm{T}_{3}$ & Carbendazim50\% WP & 0.3 & 16.63 & 19.73 & 25.61 & 4.57 & 5.19 & 5.21 \\
\hline $\mathrm{T}_{4}$ & Wettable sulphur $80 \%$ WP & 0.1 & 18.73 & 23.42 & 29.10 & 4.73 & 5.37 & 5.42 \\
\hline $\mathrm{T}_{5}$ & Wettable sulphur $80 \%$ WP & 0.2 & 16.19 & 20.88 & 26.45 & 4.70 & 5.30 & 5.36 \\
\hline $\mathrm{T}_{6}$ & Wettable sulphur $80 \%$ WP & 0.3 & 16.26 & 19.60 & 23.61 & 4.54 & 5.21 & 5.26 \\
\hline $\mathrm{T}_{7}$ & Tebuconazole 50\%+Trifloxystrobin $25 \%$ WG & 0.1 & 16.67 & 21.97 & 23.23 & 4.60 & 5.31 & 5.35 \\
\hline $\mathrm{T}_{8}$ & Tebuconazole 50\%+Trifloxystrobin $25 \%$ WG & 0.2 & 15.33 & 20.40 & 22.71 & 4.55 & 5.13 & 5.20 \\
\hline $\mathrm{T}_{9}$ & Tebuconazole 50\%+Trifloxystrobin $25 \%$ WG & 0.3 & 14.56 & 19.30 & 21.80 & 4.32 & 4.68 & 4.78 \\
\hline $\mathrm{T}_{10}$ & Carbendazim 12\% + Mancozeb 63\% WP & 0.1 & 16.21 & 20.67 & 23.63 & 4.58 & 5.05 & 5.13 \\
\hline $\mathrm{T}_{11}$ & Carbendazim 12\% + Mancozeb 63\% WP & 0.2 & 15.41 & 20.31 & 22.88 & 4.47 & 4.93 & 5.07 \\
\hline $\mathrm{T}_{12}$ & Carbendazim 12\% + Mancozeb 63\% WP & 0.3 & 15.25 & 19.32 & 21.97 & 4.33 & 4.71 & 4.85 \\
\hline $\mathrm{T}_{13}$ & Hexaconazole 5\% EC & 0.1 & 17.37 & 21.24 & 25.35 & 4.57 & 5.13 & 5.21 \\
\hline $\mathrm{T}_{14}$ & Hexaconazole 5\% EC & 0.2 & 17.31 & 22.30 & 23.52 & 4.51 & 5.10 & 5.16 \\
\hline $\mathrm{T}_{15}$ & Hexaconazole 5\% EC & 0.3 & 14.60 & 21.17 & 23.35 & 4.45 & 4.83 & 4.98 \\
\hline $\mathrm{T}_{16}$ & Difenconazole $25 \%$ EC & 0.1 & 17.45 & 21.71 & 24.28 & 4.62 & 5.18 & 5.26 \\
\hline $\mathrm{T}_{17}$ & Difenconazole $25 \%$ EC & 0.2 & 17.09 & 21.27 & 23.96 & 4.53 & 5.16 & 5.22 \\
\hline $\mathrm{T}_{18}$ & Difenconazole $25 \%$ EC & 0.3 & 15.63 & 20.80 & 23.79 & 4.45 & 4.99 & 5.13 \\
\hline $\mathrm{T}_{19}$ & Mancozeb 75\% WP & 0.1 & 17.38 & 21.08 & 25.27 & 4.61 & 5.22 & 5.29 \\
\hline $\mathrm{T}_{20}$ & Mancozeb 75\% WP & 0.2 & 15.90 & 21.32 & 25.04 & 4.59 & 5.07 & 5.17 \\
\hline $\mathrm{T}_{21}$ & Mancozeb 75\% WP & 0.3 & 14.87 & 19.47 & 22.81 & 4.47 & 4.98 & 5.09 \\
\hline \multirow[t]{3}{*}{$\mathrm{T}_{22}$} & Control (Water spray) & control & 20.30 & 24.94 & 30.62 & 4.85 & 5.47 & 5.94 \\
\hline & & S.Em \pm & 0.92 & 0.69 & 0.70 & 0.09 & 0.03 & 0.13 \\
\hline & & $\begin{array}{l}\mathbf{C D} @ \\
\mathbf{P}=\mathbf{0 . 0 5}\end{array}$ & 2.62 & 1.96 & 2.01 & 0.25 & 0.08 & 0.36 \\
\hline
\end{tabular}

$\mathrm{Tr}=$ Treatment; Figures in the parenthesis are arc sine transformed values 
Table.3 Effect of powdery mildew effective fungicides on cocoon and silk parameters of silkworm (Bombyx mori L.)

\begin{tabular}{|c|c|c|c|c|c|c|c|c|c|}
\hline $\mathbf{T r}$ & $\begin{array}{l}\text { Conc } \\
\text {. (\%) }\end{array}$ & $\begin{array}{l}\text { Average } \\
\text { Pupal } \\
\text { weight (g) }\end{array}$ & $\begin{array}{c}\text { Average } \\
\text { Cocoon } \\
\text { weight } \\
\text { (g) }\end{array}$ & $\begin{array}{l}\text { Average } \\
\text { Shell } \\
\text { weight } \\
\text { (g) }\end{array}$ & $\begin{array}{l}\text { Average Shell } \\
\text { ratio } \\
(\%)\end{array}$ & $\begin{array}{l}\text { Cocoon } \\
\text { yield/1dfl } \\
(\mathrm{g})\end{array}$ & $\begin{array}{c}\text { Average } \\
\text { filament } \\
\text { length (m) }\end{array}$ & $\begin{array}{c}\text { Average } \\
\text { filament } \\
\text { weight (g) }\end{array}$ & Denier (\%) \\
\hline $\mathrm{T}_{1}$ & 0.1 & 8.40 & 11.37 & 2.95 & $26.12(30.73)$ & 398.34 & 857.02 & 0.20 & 2.14 \\
\hline $\mathrm{T}_{2}$ & 0.2 & 6.87 & 7.23 & 1.81 & $25.03(30.02)$ & 253.05 & 813.82 & 0.21 & 2.37 \\
\hline $\mathrm{T}_{3}$ & 0.3 & 6.60 & 6.74 & 1.44 & $21.36(27.52)$ & 235.90 & 763.17 & 0.21 & 2.48 \\
\hline $\mathrm{T}_{4}$ & 0.1 & 8.29 & 11.22 & 2.93 & $26.11(30.73)$ & 392.70 & 820.58 & 0.21 & 2.27 \\
\hline $\mathrm{T}_{5}$ & 0.2 & 6.87 & 7.69 & 2.01 & $26.10(30.72)$ & 269.15 & 792.45 & 0.21 & 2.42 \\
\hline $\mathrm{T}_{6}$ & 0.3 & 6.33 & 6.83 & 1.49 & $21.81(27.84)$ & 239.05 & 786.15 & 0.22 & 2.51 \\
\hline $\mathrm{T}_{7}$ & 0.1 & 7.96 & 9.77 & 1.94 & $19.85(26.45)$ & 341.95 & 785.25 & 0.22 & 2.52 \\
\hline $\mathrm{T}_{8}$ & 0.2 & 5.74 & 7.09 & 1.35 & $19.04(25.87)$ & 248.15 & 730.57 & 0.21 & 2.57 \\
\hline $\mathrm{T}_{9}$ & 0.3 & 5.62 & 6.93 & 1.31 & $18.90(25.77)$ & 242.55 & 657.67 & 0.21 & 2.87 \\
\hline $\mathrm{T}_{10}$ & 0.1 & 7.20 & 8.95 & 1.75 & $19.55(26.24)$ & 312.21 & 783.45 & 0.20 & 2.35 \\
\hline $\mathrm{T}_{11}$ & 0.2 & 5.93 & 7.33 & 1.40 & $19.09(25.09)$ & 256.55 & 776.93 & 0.21 & 2.48 \\
\hline $\mathrm{T}_{12}$ & 0.3 & 5.76 & 7.08 & 1.32 & $18.64(25.57)$ & 247.80 & 741.38 & 0.22 & 2.68 \\
\hline $\mathrm{T}_{13}$ & 0.1 & 7.83 & 9.50 & 1.54 & $16.21(23.74)$ & 332.50 & 754.65 & 0.20 & 2.38 \\
\hline $\mathrm{T}_{14}$ & 0.2 & 6.40 & 7.61 & 1.21 & $15.90(23.50)$ & 266.35 & 745.87 & 0.20 & 2.45 \\
\hline $\mathrm{T}_{15}$ & 0.3 & 6.00 & 6.69 & 0.69 & $10.31(18.73)$ & 234.15 & 682.65 & 0.19 & 2.57 \\
\hline $\mathrm{T}_{16}$ & 0.1 & 7.70 & 9.32 & 1.62 & $17.38(24.64)$ & 326.20 & 773.55 & 0.21 & 2.44 \\
\hline $\mathrm{T}_{17}$ & 0.2 & 6.37 & 7.52 & 1.15 & $15.29(23.02)$ & 263.20 & 753.30 & 0.22 & 2.59 \\
\hline $\mathrm{T}_{18}$ & 0.3 & 6.30 & 7.15 & 0.85 & $11.88(20.16)$ & 250.25 & 723.60 & 0.22 & 2.71 \\
\hline $\mathrm{T}_{19}$ & 0.1 & 6.97 & 8.75 & 1.78 & $20.34(26.80)$ & 306.25 & 820.58 & 0.21 & 2.31 \\
\hline $\mathrm{T}_{20}$ & 0.2 & 6.09 & 7.19 & 1.10 & $15.29(23.02)$ & 250.81 & 792.45 & 0.21 & 2.43 \\
\hline $\mathrm{T}_{21}$ & 0.3 & 6.17 & 7.13 & 0.76 & $10.65(19.04)$ & 249.55 & 776.93 & 0.22 & 2.58 \\
\hline $\mathrm{T}_{22}$ & contr & 8.61 & 11.80 & 3.15 & $26.69(31.10)$ & 413.00 & 936.45 & 0.25 & 2.30 \\
\hline \multicolumn{2}{|c|}{ S.Em \pm} & 0.12 & 0.42 & 0.07 & 1.13 & 0.38 & 15.69 & 0.009 & 0.11 \\
\hline \multicolumn{2}{|c|}{$\begin{array}{l}\text { CD @ } \\
P=0.05\end{array}$} & 0.33 & 1.19 & 0.20 & 3.21 & 1.08 & 44.72 & 0.026 & 0.32 \\
\hline
\end{tabular}

$\operatorname{Tr}=$ Treatment; Figures in the parenthesis are arc sine transformed values 
The minimum larval length of $4.32 \mathrm{~cm}$ was observed in tebuconazole 50\%+ trifloxystrobin $25 \% \mathrm{WG}$ at $0.3 \%$ followed by $4.33 \mathrm{~cm}$ in carbendazim $12 \%+$ mancozeb $63 \% \mathrm{WP}$ at $0.3 \%$, hexaconazole $5 \% \mathrm{EC}$ at $0.3 \%$ and difenconazole $25 \% \mathrm{EC}$ at $0.3 \%$ recorded $4.45 \mathrm{~cm}$ and $4.47 \mathrm{~cm}$ in case of mancozeb $75 \% \mathrm{WP}$ at $0.3 \%$ (Table 2).

At $5^{\text {th }}$ instar, $6^{\text {th }}$ day, it was found that there was no significant difference between control treatment and carbendazim 50\% WP at $0.1 \%$ for larval length with $5.47 \mathrm{~cm}$ and $5.45 \mathrm{~cm}$ respectively and these were significantly superior over other treatments. The minimum larval length of $4.68 \mathrm{~cm}$ was recorded in tebuconazole $50 \%$ +trifloxystrobin $25 \%$ WG at $0.3 \%$ followed by carbendazim $12 \%+$ mancozeb $63 \%$ WP $(4.71 \mathrm{~cm})$ at $0.3 \%$. The highest larval length $5.94 \mathrm{~cm}$ was observed in control treatment which was on par with carbendazim 50\% WP $(5.73 \mathrm{~cm})$ treated at $0.1 \%$ which was significantly superior over other treatments during $5^{\text {th }}$ instar, $7^{\text {th }}$ day. The minimum larval length of $4.78 \mathrm{~cm}$ was noticed in tebuconazole 50\%+trifloxystrobin 25\% WG at $0.3 \%$ followed by carbendazim $12 \%+$ mancozeb $63 \%$ WP $(4.85 \mathrm{~cm})$ at $0.3 \%$ and hexaconazole $5 \% \mathrm{EC}(4.98 \mathrm{~cm})$ at $0.3 \%$.

\section{Fungicide residue effect on cocoon quality}

The cocoon weight was maximum $(11.80 \mathrm{~g})$ in control treatment which is on par with carbendazim 50\% WP $(11.37 \mathrm{~g})$ at $0.1 \%$ which was at par with followed by wettable sulphur $80 \%$ WP (11.22) at $0.1 \%$ but all other treatments shown significant effect on cocoon weight. The minimum cocoon weight of $6.69 \mathrm{~g}$ was noticed in hexaconazole $5 \% \mathrm{EC}$ treated at $0.3 \%$ as indicated in Table 3 .

The pupal weight was maximum $(8.65 \mathrm{~g})$ in control treatment which is on par with carbendazim 50\% WP $(8.40 \mathrm{~g})$ treated at $0.1 \%$ followed by wettable sulphur $80 \%$ WP
$(8.29 \mathrm{~g})$ at $0.1 \%$. The next best pupal weight of $7.96 \mathrm{~g}$ was observed in tebuconazole $50 \%$ +trifloxystrobin $25 \% \mathrm{WG}$ at $0.1 \%$. The minimum pupal weight of $5.62 \mathrm{~g}$ was noticed in tebuconazole 50\%+trifloxystrobin 25\% WG at $0.3 \%$ which is closely followed by tebuconazole $50 \%+$ trifloxystrobin $25 \%$ WG $(5.74 \mathrm{~g})$ at $0.2 \%$ and carbendazim $12 \%+$ mancozeb 63\% WP (5.76g) at $0.3 \%$ (Table 3 ).

The maximum shell weight $(3.15 \mathrm{~g})$ was recorded in control treatment which is on par with carbendazim 50\% WP $(2.95 \mathrm{~g})$ treated at $0.1 \%$. The next best treatment was recorded in wettable sulphur $80 \%$ WP with $(2.93 \mathrm{~g})$ at $0.1 \%$. The minimum shell weight $(0.69 \mathrm{~g})$ was recorded in hexaconazole 5\% EC at $0.3 \%$ which was on par with mancozeb $75 \%$ WP $(0.76 \mathrm{~g})$ at $0.3 \%$, followed by difenconazole $25 \%$ EC with $(0.85 \mathrm{~g})$ at $0.3 \%$ (Table 3$)$.

The findings of the present investigation are in accordance with Chandru et al., (1995) who reported that the beneficial effects of the carbendazim at 2 and 3 per cent concentration with respect to larval, cocoon, shell weight and cocoon to shell ratio.

Similar results were obtained by Govindaiah et al., (1994) who reported that feeding the silkworm with leaves treated by fungicides viz., carbendazim, dinocap, wettable sulphur chlorothalonil, mancozeb and captafol immediately after three days after spraying had no effect on silkworm and there was no significant difference in mortality percentage, weight of larvae, cocoon weight, shell weight, cocoon yield and silk ratio between control and treatments.

The maximum shell ratio of 26.69 per cent was observed in control treatment which is on par with carbendazim 50\% WP (26.12\%) treated at $0.1 \%$ and wettable sulphur $80 \% \mathrm{WP}$ $(26.11 \%)$ at $0.1 \%$, followed by wettable sulphur $80 \%$ WP $(26.10 \%)$ at $0.2 \%$ and 
carbendazim $50 \%$ WP $(25.03 \%)$ treated at $0.2 \%$. The minimum shell ratio was recorded in hexaconazole $5 \% \mathrm{EC}(10.31 \%)$ at $0.3 \%$ which was on par with mancozeb $75 \%$ WP $(10.65 \%)$ treated at $0.3 \%$ and difenconazole $25 \%$ EC (11.88\%) at $0.3 \%$ (Table 3 ).

The maximum cocoon yield of $413.00 \mathrm{~g}$ was recorded in control treatment which is statistically significant over other treatments.

The next best cocoon yield was recorded in carbendazim $50 \%$ WP with $398.34 \mathrm{~g}$ at $0.1 \%$. The lowest cocoon yield was recorded in hexaconazole $5 \% \mathrm{EC}$ at 0.3 per cent with $234.15 \mathrm{~g}$

\section{Fungicide residue effect on silk quality}

The maximum filament length of $936.45 \mathrm{~m}$ was recorded in control treatment which was significantly superior over other treatments. The next best was noticed in carbendazim $50 \%$ WP $(857.02 \mathrm{~m})$ treated at $0.1 \%$, followed by wettable sulphur $80 \%$ WP $(820.58 \mathrm{~m})$ at $0.1 \%$ and $813.82 \mathrm{~m}$ in carbendazim $50 \% \mathrm{WP}$ at $0.2 \%$. The lowest filament length of $657.67 \mathrm{~m}$ was recorded in tebuconazole $50 \%+$ trifloxystrobin $25 \% \mathrm{WG}$ at $0.3 \%$ which was significantly less when compared to other treatments as shown in Table 3.

The filament weight was maximum $(0.25 \mathrm{~g})$ in control treatment which was significantly superior over other treatments. There was no significant difference in Wettable sulphur $80 \%$ WP at $0.3 \%$, tebuconazole $50 \%+$ trifloxystrobin $25 \% \mathrm{WG}$ at $0.3 \%$, carbendazim 12\%+ mancozeb $63 \% \mathrm{WP}$ at $0.3 \%$, difenconazole $25 \%$ EC at $0.2 \%$, difenconazole $25 \% \mathrm{EC}$ at $0.3 \%$ and mancozeb $75 \% \mathrm{WP}$ at $0.3 \%$ with $(0.22 \mathrm{~g})$ and rest of the other treatments were on par with each other with $0.21 \mathrm{~g}$. The minimum filament weight $(0.19 \mathrm{~g})$ was recorded in hexaconazole $5 \% \mathrm{EC}$ at $0.3 \%$ (Table 3 ).
The effect of fungicides on denier was significant. The larvae administered with leaves treated with carbendazim 50\% WP at $0.1 \%$ recorded the lowest denier of (2.14) which was significantly superior over control (2.40). The fungicidal treatments viz., wettable sulphur $80 \% \mathrm{WP}$ at $0.1 \%$, mancozeb $75 \% \mathrm{WP}$ at $0.1 \%$, carbendazim $12 \%+$ mancozeb $63 \%$ WP at $(0.1 \%$, carbendazim $50 \%$ WP at $0.2 \%$ and hexaconazole 5\% EC affected little on denier of 2.27, 2.31, 2.35, 2.37 and 2.38 respectively which were on par with control treatment (2.40). However, the fungicide tebuconazole $50 \%+$ trifloxystrobin $25 \%$ WG at $0.3 \%$ failed to improve the denier as they recorded 2.83 and was on par with carbendazim $12 \%+$ mancozeb $63 \%$ WP (2.68) treated at $0.3 \%$ and difenconazole $25 \% \mathrm{EC}$ (2.59) at $(0.3 \%)$ followed by mancozeb $75 \%$ WP (2.58) at $0.3 \%$, whereas, hexaconazole $5 \%$ EC and tebuconazole 50\%+ trifloxystrobin $25 \% \mathrm{WG}$ showed 2.57 at $0.3 \%$ followed by wettable sulphur $80 \%$ WP (2.51) at $0.3 \%$ (Table 3 ).

\section{References}

Aherkar, S. K., Thakare, H. S., Gawanda, R. B. and Sarnik, D. N., 1995, Effect of fungicide treated leaves on silkworm larvae, Bombyx mori L. Pestology. 12: $15-19$.

Chandra, A. K., Saha, R. K., Bhattacharya, J. and Krishnan, N., 1995, Efficacy of carbendazim as an anti-microsporidial agent and its influence on the growth and cocoon characters of silkworm, Bombyx mori L. Int. J. Trop. Insect Sci., 16(2):233-235.

Chattopadhyay, S., Akhter, A. K., Gandhi, D. S., Das, N. K., Aggarwal, R. K., Tapas, K. B., Sarkar, A. and Bajpai, A. K., 2011, Association of leaf micromorphological characters with powdery mildew resistance in field-grown mulberry (Morus spp.) germplasm. $A o B$ 
PLANTS, doi:10.1093/aobpla/plr002.

Dutta, S. K., Maji, M. D., Gosh, A., Roy Chowdhury, S., Choudhuri, B., Majumdar, M. K. and Bindroo, B., 2011, Survey on disease severity of mulberry (Morus alba L.) in different seasons of Aizawl districts (Mizoram). J.Crop. Weed. 7(2): 253-253.

Gayathri, M. and Rajendra, P. P., 2011, Effect of fungicides on Phyllactinia corylea conidial germination and on silkworm. Indian J. Entomol., 73(2): 143-145.

Govindaiah, Gunasekhar, V., Puttaswamy, G. and Thiagarajan, V., 1994, Field evaluation of fungicides against Phyllactinia corylea causing powdery mildew in mulberry (Morus alba L.). Indian J. Seric., 33:160-162.
Sikdar, A.K., Samson, M.V., Madhava Rao, Y.R., Murthuza Baig and Nataraju, B., 1979, Effect of feeding leaf spot affected andsystemic fungicide sprayed leaves of mulberry (Morus indica L)on silk worm (Bombyx mori L.). Indian Journal of Sericulture, 18(1): 73-77.

Sukumar, J. and Ramalingam, A.L., 1989, Epidemiology of Cercospora moricola leaf spot disease of mulberry. III Conidial dispersal and disease incidence. Sericologia, 29: 533-539.

Teotia, R.S. and Sen, S.K., 1994, Mulberry diseases in India and their control. Sericologia, 34: 1-18.

\section{How to cite this article:}

Manjunatha, S.E., V.B. Sanath Kumar and Kiran Kumar, N. 2017. Toxicological Studies of Mulberry Powdery Mildew Effective Fungicide Residues on Growth and Development of Silkworm (Bombyx mori L.), Cocoon and Silk Quality Parameters. Int.J.Curr.Microbiol.App.Sci. 6(11): 708-716. doi: https://doi.org/10.20546/ijcmas.2017.611.083 